\title{
Síndrome de Behçet e Policondrite Recidivante: Síndrome MAG/C
}

\author{
Behçet's Syndrome and Relapsing Polychondritis: \\ MAGIC Syndrome
}

\author{
Fabrício de Souza Neves ${ }^{(1)}$, Deborah Pereira Gonçalves ${ }^{(1)}$, Laís V. Lage ${ }^{(2)}$, \\ Célio Roberto Gonçalves ${ }^{(2)}$
}

\section{RESUMO}

Relatamos aqui o caso de um paciente masculino de 30 anos de idade, sexo masculino, com achados clínicos típicos da síndrome de Behçet (SB) e da policondrite recidivante (PR) sem, no entanto, preencher completamente os critérios de classificação para nenhuma das entidades isoladamente. O termo síndrome MAGIC (Mouth And Genital ulcers with Inflamed Cartilage) tem sido usado para classificar pacientes semelhantes. Uma breve revisão dos casos previamente relatados também é apresentada.

Palavras-chave: síndrome de Behçet, policondrite recidivante, vasculite.

\section{INTRODUÇÃO}

A síndrome de Behçet (SB) e a policondrite recidivante (PR) são afecções inflamatórias multissistêmicas raras, de provável natureza auto-imune, que apresentam manifestações clínicas razoavelmente específicas permitindo seu diagnóstico através de conjuntos de critérios bem definidos. A ocorrência de manifestações das duas entidades no mesmo paciente foi relatada pela primeira vez por Firestein et al ${ }^{(1)}$, em 1985, numa série de cinco casos, propondo a esta associação a denominação síndrome MAGIC (Mouth And Genital ulcers with Inflamed Cartilage) e sugerindo a existência de um mecanismo patogênico comum às duas entidades. Apresentamos nesta comunicação a descrição de um caso brasileiro desta rara associação.

\begin{abstract}
We describe a 30 years old man with typical features of both Behçet's syndrome and relapsing polychondritis but neither complete classification criteria. The term MAGIC syndrome (Mouth And Genital ulcers with Inflamed Cartilage) has been used to describe similarly affected patients. We present a brief review of previously reported cases.
\end{abstract}

Keywords: Behcet's syndrome, polychondritis, relapsing, vasculitis.

\section{RELATO DE CASO}

Um paciente do sexo masculino, de 30 anos, em acompanhamento ambulatorial regular desde junho de 2002 com história de úlceras aftosas orais de repetição, associadas à pseudofoliculite e eritema nodoso em perna direita, desde fevereiro de 2001. Apresentou orquiepidimite em setembro de 2001 e trombose venosa profunda em perna direita em outubro de 2001, sendo iniciada anticoagulação com varfarina e encaminhado ao Serviço de Reumatologia com a hipótese diagnóstica de SB. Negava úlceras genitais, não foi encontrado acometimento ocular em avaliação oftalmológica e o teste de patergia foi negativo. Também não apresentava sintomas gastrintestinais. Tinha provas de função hepática, renal, hematimetria e

Hospital das Clínicas da Faculdade de Medicina da Universidade de São Paulo (HC-USP). Recebido em 05/12/05. Aprovado, após revisão, em 28/03/06.

1. Médico Residente da Disciplina de Reumatologia do HC-USP.

2. Médico Assistente da Disciplina de Reumatologia do HC-USP.

Endereço para correspondência: Fabrício de Souza Neves, Av. Dr. Altino Arantes 742, ap 84, Vila Clementino, São Paulo, CEP 04042-003, SP,

e-mail: nevesfab bol.com.br 
eletrólitos normais. Velocidade de hemossedimentação (VHS) era $28 \mathrm{~mm} / \mathrm{h}$ (Westergren) e Proteína-C reativa (PCR) era $36,0 \mathrm{mcg} / \mathrm{ml}$ (Nefelometria, normal até 5 $\mathrm{mcg} / \mathrm{ml}$ ). Pesquisa de Fator Antinuclear, anticorpos anticardiolipina e de anticoagulante lúpico foram negativos. Os níveis das proteínas $\mathrm{C}, \mathrm{S}$ e de antitrombina III eram normais. Terapia com prednisona $20 \mathrm{mg}$ por dia e colchicina foi iniciada, sendo também mantida a anticoagulação com varfarina. Embora tenha evoluído inicialmente com melhora clínica e normalização de VHS e PCR, persistiu com surtos recorrentes de atividade caracterizados por úlceras orais e eritema nodoso. Azatioprina foi iniciada em outubro de 2002, sendo substituída por ineficácia, em abril de 2003, por talidomida, e esta por clorambucil, em janeiro de 2004, ao qual foram associados pentoxifilina e penicilina benzatina, também com pouca eficácia.

Em agosto de 2005, apresentou-se com novo eritema nodoso (Figura 1), úlceras aftosas orais, aumento de PCR $(37,4 \mathrm{mcg} / \mathrm{ml}$ por nefelometria) e VHS 8 (Westergren), mas também com dor, eritema e aumento de volume em pavilhão auricular esquerdo poupando o lóbulo (Figura 2). Referia que esta sintomatologia ocorrera também na orelha direita duas semanas antes, com melhora espontânea. A dose de prednisona foi aumentada para $40 \mathrm{mg}$ por dia, havendo melhora da inflamação auricular após uma semana.

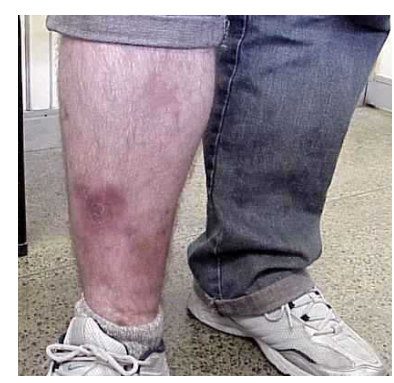

Figura 1 - Eritema nodoso em atividade na perna direita.

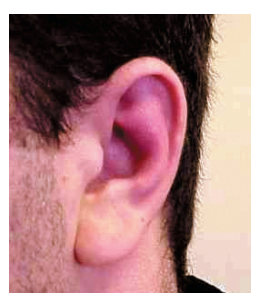

Figura 2 - Inflamação do pavilhão auricular esquerdo, poupando lóbulo.

\section{DISCUSSÃO}

A SB pode ser diagnosticada através dos critérios propostos pelo International Study Group for Behçet's Disease em 1990 (2): (1) ulcerações orais recorrentes devem obrigatoriamente estar presentes, associadas a quaisquer dois dos seguintes critérios: (2) ulceração genital recorrente; (3) lesões oculares, incluindo uveíte anterior, uveíte posterior, células no vítreo ou vasculite retiniana; (4) lesões cutâneas, incluindo eritema nodoso, pseudofoliculite ou lesões acneiformes; (5) teste positivo de patergia. Já a PRé diagnosticada pelos critérios propostos por $\mathrm{McAdam}^{(3)}$, em 1976, quando três ou mais dos seguintes estão presentes: (1) condrite auricular bilateral; (2) poliartrite inflamatória soronegativa não-erosiva; (3) condrite nasal; (4) inflamação ocular, incluindo conjuntivite, ceratite, esclerite, episclerite ou uveíte; (5) condrite do trato respiratório; (6) lesão audiovestibular. Em 1979, Damiani e Levine ${ }^{(4)}$ sugeriram que o diagnóstico de PR também poderia ser feito na presença de ao menos um dos critérios de McAdam com comprovação histológica, ou condrite em duas regiões anatômicas distintas com resposta a corticosteróides ou dapsona.

Firestein et $a{ }^{1)}$ foram os primeiros a observar que muitas manifestações clínicas menos específicas eram comuns às duas entidades, sendo descritas em um grupo de cinco pacientes apresentando ambos: úlceras orogenitais e inflamação auricular. Para estes autores, condrite é manifestação altamente sugestiva de PR, enquanto úlceras orogenitais o são de SB. Eritema nodoso e pseudofoliculite, embora mais comuns na $\mathrm{SB}$, também ocorrem na $\mathrm{PR}$, enquanto tromboses, inflamação ocular, aneurismas arteriais, artrites e lesões de natureza vasculítica em sistema nervoso central (SNC) e trato gastrintestinal são igualmente freqüentes ${ }^{(1)}$. Posteriormente, outros autores descreveram casos isolados semelhantes, empregando o acróstico MAGIC proposto por Firestein ${ }^{(1)}$ para classificá-los. Nesses casos, úlceras orogenitais e condrite auricular coexistiam com outras manifestações típicas, algumas vezes sem necessariamente preencher os critérios de classificação necessários para SB ou $\mathrm{PR}^{(5-10)}$, assim como no caso aqui apresentado. Uma breve revisão das características clínicas desses casos é apresentada na Tabela 1.

No caso apresentado, as opções terapêuticas foram sendo sucessivamente tentadas baseando-se no diagnóstico inicial de SB: colchicina e talidomida podem ser usados na prevenção das recidivas de lesões mucocutâneas (os dois avaliados em ensaios clínicos controlados), embora apresentando resultados apenas modestos ${ }^{(11,12)}$. Terapia imunossupressora foi iniciada com azatioprina, sendo esta a medicação mais adequadamente estudada na SB. Ela efetivamente demonstrou melhorar o prognóstico da função ocular em longo prazo $^{(13)}$, com sua eficácia avaliada em ensaio clínico controlado. Nesse estudo, além da redução significativa na ocorrência de episódios de uveíte ao longo de dois anos de seguimento, houve também redução na freqüência de úlceras orais, genitais e artrites no grupo em uso de azatioprina ${ }^{(14)}$. Redução na freqüência de episódios de artrites e lesões mucocutâneas também foi demonstrada 
com o uso de penicilina benzatina, num estudo randomizado e controlado que comparou colchicina isolada contra a associação de colchicina com penicilina benzatina (antígenos estreptocócicos são considerados potenciais desencadeadores da SB $)^{(15,16)}$. O uso de agentes biológicos anti-TNF é descrito com sucesso em séries e relatos de casos, tanto para $\mathrm{SB}^{(17)}$ como para $\mathrm{PR}^{(18)}$. A experiência terapêutica com a síndrome MAGIC se resume aos relatos de casos, com sucesso descrito para o uso de dapsona ${ }^{(5)} \mathrm{e}$ pentoxifilina ${ }^{(8)}$.

Embora seja relativamente comum a associação da PR com outras doenças auto-imunes ${ }^{(19)}$, Firestein et a $\left(^{(1)}\right.$ valorizaram a existência de diversas manifestações clínicas compartilhadas entre SB e PR para sugerir a existência um mecanismo fisiopatogênico comum às duas entidades que caracterizaria a nova síndrome. Auto-imunidade contra a elastina (um componente da cartilagem que também é encontrado na pele) já foi considerada suspeita, devido a estudos de microscopia eletrônica que descreveram materiais eletrodensos em torno de fibras elásticas de cartilagens auriculares de pacientes com $\mathrm{PR}^{(20)}$. Tal hipótese, porém, ainda não foi averiguada. Se a síndrome MAGIC consiste uma nova entidade isolada, uma associação fortuita entre duas síndromes raras ou se é uma sobreposição, na qual SB e PR são pontos diferentes de um mesmo espectro fisiopatogênico, ainda é uma questão sem resposta definitiva.

TABELA 1

Características CLÍNICAS DESCRITAS NA SÍNDROME MAGIC

\begin{tabular}{|c|c|c|c|c|c|c|c|c|c|c|c|c|}
\hline $\begin{array}{c}\text { Paciente } \\
\text { (Referência) }\end{array}$ & $\begin{array}{c}1 \\
(1)\end{array}$ & $\begin{array}{c}2 \\
(1)\end{array}$ & $\begin{array}{c}3 \\
(1)\end{array}$ & $\begin{array}{c}4 \\
(1)\end{array}$ & $\begin{array}{c}5 \\
(1)\end{array}$ & $\begin{array}{c}6 \\
(5)\end{array}$ & $\begin{array}{c}7 \\
(6)\end{array}$ & $\begin{array}{l}8 \\
(7)\end{array}$ & $\begin{array}{c}9 \\
(8)\end{array}$ & $\begin{array}{l}10 \\
(9)\end{array}$ & $\begin{array}{c}11 \\
(10)\end{array}$ & $\begin{array}{c}12 \\
\text { (atual) }\end{array}$ \\
\hline Idade e sexo & $25, F$ & $10, F$ & $59, M$ & $26, F$ & $39, F$ & $42, M$ & $22, M$ & $25, M$ & $39, F$ & $24, M$ & $38, M$ & $30, M$ \\
\hline Tempo evolução & 9 & 5 & 6 & 7 & 14 & 7 & 4 & 1 & 2 & 7 & 10 & 5 \\
\hline Úlceras orais & - & $\bullet$ & - & - & - & $\bullet$ & - & $\bullet$ & $\bullet$ & $\bullet$ & $\bullet$ & $\bullet$ \\
\hline Úlceras genitais & - & $\bullet$ & $\bullet$ & - & - & & $\bullet$ & $\bullet$ & $\bullet$ & $\bullet$ & $\bullet$ & \\
\hline Uveíte & - & & & • & & & & - & & & & \\
\hline
\end{tabular}

Vasculite retina

\begin{tabular}{|c|c|c|c|c|c|c|c|c|c|c|c|c|}
\hline \multicolumn{7}{|l|}{ Eritema nodoso } & \multicolumn{5}{|c|}{ - } & \multirow{2}{*}{$\begin{array}{l}\bullet \\
\bullet \bullet\end{array}$} \\
\hline Pseudofoliculite & $\bullet$ & - & & & & $\bullet$ & $\bullet$ & & & $\bullet$ & $\bullet$ & \\
\hline Patergia & & & & & & & - & & & & & \\
\hline Cond. Aur. & $\bullet$ & $\bullet$ & $\bullet$ & $\bullet$ & $\bullet$ & $\bullet$ & $\bullet$ & $\bullet$ & $\bullet$ & $\bullet$ & $\bullet$ & $\bullet$ \\
\hline Poliartrite & $\bullet$ & $\bullet$ & - & - & $\bullet$ & $\bullet$ & - & $\bullet$ & $\bullet$ & $\bullet$ & & \\
\hline Condrite nasal & - & $\bullet$ & $\bullet$ & $\bullet$ & - & & - & $\bullet$ & & & & \\
\hline Ocular - outros & & • & - & & $\bullet$ & $\bullet$ & & & - & - & - & \\
\hline Cond. Resp. & & & & $\bullet$ & & & & & & & & \\
\hline Audiovestibular & & & & $\bullet$ & - & & & - & & & & \\
\hline Vasc. cutânea & & $\bullet$ & & & $\bullet$ & & & $\bullet$ & & & & \\
\hline Tromboses & - & & $\bullet$ & $\bullet$ & & & & & & $\bullet$ & & $\bullet$ \\
\hline Gastrintestinal & & & $\bullet$ & & & $\bullet$ & & & $\bullet$ & & & \\
\hline SNC & & & & - & & & & & & & $\bullet$ & \\
\hline Orquiepididimite & & - & & & & & & & & & & - \\
\hline
\end{tabular}

$M=$ masculino; F = feminino; Cond. Aur. = condrite auricular bilateral; Ocular - outros = esclerite, episclerite, conjuntivite, ceratite; Cond. Resp. = condrite do trato respiratório; Vasc. Cutânea = vasculite cutânea; SNC = sitema nervoso central. 0 sinal “+" indica descrição compatível com a característica clínica no caso mencionado. "Idade", expressa em anos, refere-se ao início das manifestações clínicas; “tempo de evolução", expresso em anos, refere-se ao período desde o início dos sintomas até o estabelecimento da sobreposição caracterizada como MAGIC. MAGIC: Mouth And Genital ulcers with Inflamed Cartilage. 


\section{REFERÊNCIAS}

1. Firestein GS, Guber HE, Weisman MH, Zvaifler NJ, Barber J, O'Duffy JD. Mouth and genital ulcers with inflamed cartilage: MAGIC syndrome. Five patients with features of relapsing polychondritis and Behçet's disease. Am J Med 79:65-72, 1985.

2. International Study Group for Behçet's Disease. Criteria for diagnosis of Behçet's disease. Lancet 335: 1078-80, 1990.

3. McAdam LP, O’Hanlom MA, Blestone R, Pearson CM.Relapsing polychondritis: prospective study of 23 patients and review of the literature. Medicine (Baltimore) 55:193-215, 1976.

4. Damiani JM, Levine HL. Relapsing polychondritis - report of ten cases. Laryngoscope 89: 929-44, 1979.

5. Orme RL, Nordlund JJ, Barich L, Brown T. The MAGIC syndrome (mouth and genital ulcers with inflamed cartilage). Arch Dermatol 126: 940-4, 1990.

6. Le Thi Huong D, Wechsler B, Piette JC et al. Aortic insufficiency and recurrent valve prostjesis dehiscence in MAGIC syndrome. J Rheumatol 20: 397-8, 1993.

7. Belzunegui J, Cancio J, Pego JM, Uriarte E, Iribarren JA. Relapsing polychondritis and Behçet's syndrome in a patient with HIV infection. Ann Rheum Dis 54: 780, 1995.

8. Imai $\mathrm{H}$, Motegi $\mathrm{m}$, Mizuki $\mathrm{N}$ et al. Mouth and genital ulcers with inflamed cartilage (MAGIC syndrome): a case report and literature review. Am J Med Sci 314:330-2, 1997.

9. Gamboa F, Rivera JM, Mayoral L, Grilo A. Behçet's disease and relapsing polychondritis (MAGIC syndrome) associated with antiphospolipid syndrome. Med Clin (Barc) 110: 678-9, 1998.

10. Gertner E. Severe recurrent neurologic disease in the MAGIC syndrome. J Rheumatol 31:1018-9, 2004.

11. Yurdakul S, Mat C, Tuzun Y et al. A double-blind trial of colchicine in Behçet's Syndrome. Arthritis Rheum 44: 2686-92, 2001.

12. Hamuryudan V, Mat C, Saip S et al. Thalidomide in the treatment of the mucocutaneous lesions of the Behçet syndrome: a randomized, double-blind, placebo-controlled trial. Ann Intern Med 128: 443-50, 1998.

13. Hamuryudan V, Özyazgan $\mathrm{Y}$, Hizli $\mathrm{N}$ et al. Azathioprine in Behçet's síndrome: effects on long term prognosis. Arthritis Rheum 40: 769-74, 1997.

14. Yazici H, Pazarli H, Barnes CG . A controlled trial of azathioprine in Behçet's Syndrome. N Engl J Med 322: 281-85, 1990.

15. Calguneri M, Ertenli I, Kiraz S, Erman M, Celik I. Effect of prophylactic benzathine penicillin on mucocutaneous symptoms of Behçet's Disease. Dermatology 192: 125-8, 1996.

16. Calgumeri M, Kiraz S, Ertenli I, Benekli M, Karaarslan Y, Celik I. The effect of prophylactic penicillin treatment on the course of arthritis episodes in patients with Behçet's Disease. A randomized clinical trial. Arthritis Rheum 39: 2062-5, 1996.

17. Sfikakis PP, Theodossiadis PG, Katsiari GG, Kaklamanis P, Markomichelakis NN. Effect of infliximab on sight treatening panuveitis in Behçet's disease. Lancet 358: 295-6, 2001.

18. Carter JD. Treatment of relapsing polychondritis with a TNF antagonist. JRheumatol 32:1413, 2005.

19. Trentham DE, Le CH. Relapsing polychondritis. Ann Intern Med 129: 114-22, 1998.

20. Shaul SR, Schumacher HR. Relapsing polychondritis: electron microscope study of ear cartilage. Arthritis Rheum 18: 617-25, 1975. 